\title{
Avaliação dos Habilitadores da Educação 4.0: Transformação Digital da Educação Multidisciplinar em Mudanças Climáticas
}

\section{Katyeudo Karlos de S. Oliveira ${ }^{1}$, Maria Lydia Fioravanti ${ }^{1}$, Ellen Francine Barbosa ${ }^{1}$, Ricardo André C. de Souza ${ }^{2}$}

\author{
${ }^{1}$ Universidade de São Paulo (USP) - São Carlos, SP, Brasil \\ ${ }^{2}$ Universidade Federal Rural de Pernambuco (UFRPE) - Recife, PE, Brasil \\ \{karlos_oliveira, mlfioravanti\}@usp.br, francine@icmc.usp.br \\ ricardo.souza@ufrpe.br
}

\begin{abstract}
Resumo. A educação é um meio estratégico de mitigação e adaptação humana às mudanças climáticas. Ao invés de ser tratado separadamente e ensinado e aprendido de modo tradicional, o tema 'mudanças climáticas' pode estar conectado às disciplinas básicas num paradigma de Educação 4.0. Este trabalho apresenta a especificação e avaliação de propostas de uso dos habilitadores da Educação 4.0, visando a transferência e aquisição de conhecimentos de disciplinas básicas a partir da problemática de causas e impactos das mudanças climáticas. Ainda, são discutidos os resultados da análise de 53 educadores, apontando que tais propostas têm potencial de agregar valor a estudantes e professores.
\end{abstract}

\begin{abstract}
Education is a strategic means of mitigation and human adaptation to climate change. Rather than being treated separately and taught and learned in a traditional way, the theme 'climate change' can be connected to the core subjects in an Education 4.0 paradigm. This work presents the specification and evaluation of proposals for the use of Education 4.0 drivers, aiming at the transfer and acquisition of knowledge of basic subjects from the problematic of causes and impacts of climate change. Furthermore, the results of the analysis of 53 educators are discussed, pointing out that such proposals have the potential to add value to students and teachers.
\end{abstract}

\section{Introdução}

As medidas adotadas contra a pandemia de COVID-19 produziram retração econômica e deixaram, conforme a UNESCO (2020), mais de 1 bilhão de estudantes de todos os níveis fora da sala de aula, demonstrando que os sistemas educacionais não estão preparados quando há restrição de espaço e tempo. Entretanto, também foi possível notar o impacto da ação humana no meio ambiente. Com a diminuição da presença humana, as águas dos mares ficaram mais límpidas, alguns animais raros puderam ser vistos novamente, e a redução da poluição melhorou a qualidade do ar [Rosenbloom e Markard 2020].

Segundo a Organisation for Economic Co-operation and Development [OECD 2019], a ação antrópica é um dos motivos causadores das mudanças climáticas, resultando em aquecimento global, desertificação, elevação da temperatura da água do mar, etc. A educação tem a capacidade de provocar nos estudantes uma mudança comportamental para atitudes mais responsáveis e ambientalmente sustentáveis. Entre as metas de desenvolvimento sustentável até 2030 da ONU (2015) destaca-se "melhorar a educação, aumentar a conscientização e a capacidade humana e institucional sobre mitigação, adaptação, redução de impacto e alerta precoce da mudança do clima". 
De acordo com a United Nations Educational, Scientific and Cultural Organization [UNESCO 2013], a educação, no contexto ambiental, pode exercer um triplo papel: (1) efetivar seu dever no desenvolvimento de capacidades e atitudes sociais e individuais para mitigar as mudanças climáticas e habilitar as pessoas para agir proativamente em relação ao futuro; (2) desenvolver competências, capacidades e atitudes, para adaptar as pessoas aos impactos climáticos evidentes e iminentes; e (3) estimular e reforçar o entendimento das mudanças climáticas, prevenindo as pessoas para esse cenário.

O tema das mudanças climáticas está se tornando protagonista em programas e currículos escolares de diversos países, mas ainda é discutido superficialmente e como um assunto isolado no contexto das disciplinas tradicionais [UNESCO 2013]. De fato, essa temática necessita estar mais conectada aos diversos conhecimentos de física, matemática, biologia, ciências, tecnologia, etc. [UNESCO 2016]. Porém, também é essencial uma estratégia de ensino-aprendizagem que simultaneamente seja atrativa e engaje os estudantes, proporcionando-lhes as habilidades exigidas para o aprendizado do século XXI [UNESCO 2019].

Também é importante ressaltar outro problema: os sistemas educacionais ainda são orientados por modelos introduzidos há décadas, enquanto a sociedade entra na $4^{\mathrm{a}}$ Revolução Industrial [WEF 2016], introduzindo a produção automatizada como nunca antes observado [WEF 2020]. Essas novas condições de crescimento causaram o surgimento de fortes mudanças nas habilidades necessárias para que as pessoas possam contribuir com a economia e nos modos com que trabalham, enfatizando questões acerca da adequação e aperfeiçoamento dos sistemas educacionais atuais, a chamada Educação 4.0 [WEF 2020].

Considerando o contexto discutido anteriormente, [De Sousa Oliveira e de Souza 2020] apresentam habilitadores para Transformação Digital em direção à Educação 4.0. Tais habilitadores norteiam o desenho e a implementação de estratégias para repensar o processo de ensino-aprendizagem para um público de nativos digitais, considerando os aspectos de: (i) fazer digital, por meio de conhecimentos e competências técnicas; e (ii) ser digital, por meio da incorporação de habilidades, mentalidade e atitudes digitais.

Neste sentido, usando a temática de causas e efeitos das mudanças climáticas como mote para o ensino e aprendizado de conhecimentos relacionados às disciplinas de Física e Biologia, este trabalho tem como objetivo apresentar a especificação e avaliação de uso dos habilitadores para a transformação digital em direção à Educação 4.0, apresentados por [De Sousa Oliveira e de Souza 2020].

Além desta seção introdutória, este trabalho está organizado em mais cinco seções. A Seção 2 descreve o arcabouço teórico e as etapas do método de pesquisa para o desenvolvimento do trabalho. Na Seção 3 e na Seção 4 são detalhadas as etapas do método de pesquisa e os resultados obtidos, respectivamente. Na Seção 5 é realizada uma breve análise dos resultados. Finalmente, na Seção 6, é descrita a conclusão.

\section{Arcabouço teórico e método de pesquisa}

A Educação 4.0 aborda as mudanças da educação em resposta à Quarta Revolução Industrial, em que a transformação digital está impactando o modo como o trabalho e a vida cotidiana estão se tornando constantemente mais automatizadas. O uso produtivo das tecnologias digitais é uma demanda da Quarta Revolução Industrial estabelecida na última década e que está influenciando a transição do trabalho mental humano para a inteligência artificial e a automação [Frerich et al. 2016; WEF 2020]. 
De acordo com o Fórum Econômico Mundial [WEF 2020], a Educação 4.0 deve abordar os desafios mais prementes da atualidade como, por exemplo, mudanças climáticas e degradação do ecossistema, introduzindo uma mentalidade ecológica de estudos, envolvendo pedagogias e novas teorias curriculares, com o propósito de motivar o engajamento e a ação humana na resolução de problemas ambientais.

No sistema educacional tradicional, as causas e consequências das mudanças climáticas geralmente são tratadas nos programas e currículos escolares como um tópico específico e desarticulado do ensino dos conhecimentos da maioria das disciplinas básicas. Segundo a [UNESCO 2016; Oliveira e de Souza 2020], as mudanças climáticas podem ser fortemente conectadas e direcionar experiências de ensino-aprendizagem nas diversas disciplinas escolares. Desse modo, a educação pode ser apontada como uma importante ferramenta para a prevenção e mitigação de riscos de desastres, com potencial para suscitar o interesse das gerações mais jovens sobre este problema [Gonçalves, Lopes e Reis 2019].

Segundo [De Sousa Oliveira e de Souza 2020], a transformação digital em direção à Educação 4.0 deve tratar de maneira holística os seguintes fatores que serviram para categorizar os habilitadores: uso de tecnologias digitais, considerando as especificidades dos estudantes; adoção de processos e práticas organizacionais em alinhamento com as novas relações sociais e de trabalho; equipar professores com competências digitais (fazer e ser) para tratar com estudantes nativos digitais; equipar estudantes com as habilidades técnicas, cognitivas e sociais necessárias para $\mathrm{o}$ aprendizado e trabalho do século XXI; e adoção de pedagogias inovadoras centradas na transferência e aquisição de conhecimento sob demanda para resolver um problema ou realizar uma tarefa.

Visando atingir o objetivo deste trabalho, é realizada a especificação e avaliação de uso dos habilitadores supracitados. Tais propostas procuram introduzir as causas e efeitos das mudanças climáticas no contexto das disciplinas de Biologia e Física. A Figura 1 apresenta, em notação Business Process Model and Notation (BPMN), o fluxo dos passos realizados para desenvolvimento do trabalho.

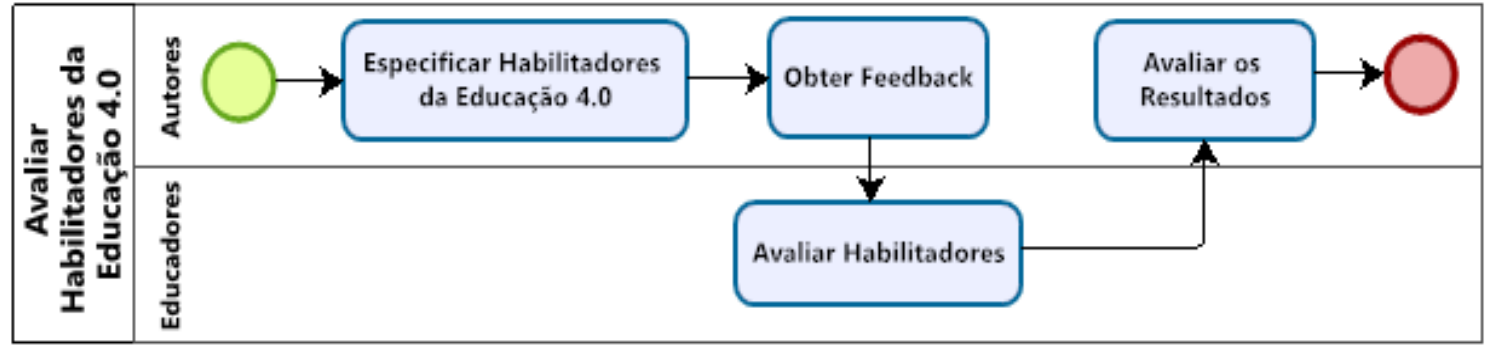

Figura 1 - Fluxo do método de pesquisa

O fluxo (pool) "Avaliar Habilitadores da Educação 4.0" é organizado em duas raias (swimlanes) para separar as atividades de responsabilidades dos atores envolvidos: o autor do trabalho e os educadores responsáveis pela avaliação dos habilitadores. $\mathrm{O}$ fluxo iniciou com a elaboração de duas propostas baseadas em alguns dos habilitadores para transformação digital em direção a Educação 4.0, com o objetivo pedagógico de tratar a multidisciplinaridade das mudanças climáticas no contexto das disciplinas de Biologia e Física. Em seguida, foi realizada a atividade "Obter feedback", que consistiu na preparação de uma apresentação, na forma de vídeo, das propostas e na elaboração e aplicação de um questionário eletrônico para avaliação destas propostas. Um conjunto de 53 educadores foram contatados por meio de diferentes canais de comunicação, como redes sociais e e-mail. Cada educador assistiu ao vídeo e preencheu o questionário 
eletrônico de avaliação, consistindo da atividade "Avaliar Habilitadores". Por fim, os resultados das avaliações foram analisados durante a atividade "Analisar Resultados".

\section{Especificar habilitadores da Educação 4.0}

Esta atividade consistiu na especificação dos habilitadores, considerando a competência da Área 1 da matriz de referência de Ciências da Natureza e suas Tecnologias do Exame Nacional do Ensino Médio (ENEM) [INEP 2012], com os respectivos objetivos: estudar o impacto das consequências das mudanças climáticas, como desmatamento e desertificação, nos biomas naturais; e estudar termologia por meio das perturbações causadas pelo aumento da temperatura do planeta ao longo do tempo. Ambas as propostas preveem a possibilidade dos estudantes trabalharem em grupo para encontrar, formular e apresentar possíveis soluções para as temáticas tratadas de maneira colaborativa. Para tanto, para cada proposta foi necessário instanciar habilitadores específicos, conforme descrito nas próximas seções.

\subsection{Proposta 1: mudanças climáticas integradas à disciplina de Biologia}

Esta especificação busca tratar sobre a preservação da biodiversidade e mensurar a degradação dos biomas causada pelas mudanças climáticas. Os educadores darão a oportunidade dos estudantes compreenderem a complexidade dos biomas brasileiros, observando a sua diversidade natural, considerando, também, as ameaças ocasionadas pela expansão econômica e por outras formas de uso e ocupação territorial. Os educadores poderão listar, juntamente com os estudantes, os tipos de biomas e, em seguida, colocá-los em equipes, atribuindo para cada uma o estudo de um dos biomas apresentados anteriormente, orientando-os que observem em cada um deles: características; região no Brasil onde se encontram; clima; fauna e flora; principais problemas de degradação e as mudanças climáticas causadas. A Tabela 1 apresenta os habilitadores da Educação 4.0 para a Proposta 1.

Tabela 1. Habilitadores da Educação 4.0 para a Proposta 1

\begin{tabular}{|c|c|}
\hline Habilitador & Descrição \\
\hline $\begin{array}{l}\text { Habilitadores } \\
\text { Tecnológicos }\end{array}$ & $\begin{array}{l}\text { Jogos Digitais: O Minecraft Education Edition }{ }^{1} \text { estimula a criatividade, a colaboração e } \\
\text { a solução de problemas através de um ambiente digital imersivo, utilizando } \\
\text { programação em blocos com desafios e estímulos para executar tarefas envolvendo } \\
\text { conceitos sobre mudanças climáticas e educação ambiental. Software Educativo: O } \\
\text { PhET }^{2} \text { permite simulaçôes de diferentes áreas do conhecimento, possui conteúdo em } \\
\text { língua portuguesa para temas como: radioatividade, frações, osmose e outros. } \\
\text { Possibilita o estudo sobre possíveis soluções de cenários de degradação ambiental e } \\
\text { futuro da Terra. }\end{array}$ \\
\hline $\begin{array}{l}\text { Habilitadores } \\
\text { Organizacionais }\end{array}$ & $\begin{array}{l}\text { Melhorar a experiência educacional com o uso de tecnologias digitais: introdução } \\
\text { de tecnologias digitais nas experiências didáticas e na rotina diária para elevar o } \\
\text { engajamento dos alunos, especialmente se aplicadas com metodologias ativas. Utilizar } \\
\text { métodos híbridos de ensino: favorecer oportunidades educacionais individuais e } \\
\text { coletivas, mitigando restrições de espaço e tempo nas interações entre os estudantes e } \\
\text { professores. }\end{array}$ \\
\hline $\begin{array}{l}\text { Competência } \\
\text { Digital Docente }\end{array}$ & $\begin{array}{l}\text { Fornecer feedback constante: discussões construtivas através de comentários, } \\
\text { sugestões, questionamentos, etc., assim, aumentando a profundidade da experiência e } \\
\text { possibilitando a correção de rotas de aprendizagem com base em evidências. Saber } \\
\text { qual tecnologia utilizar de acordo com a necessidade: gerenciar o uso dos } \\
\text { habilitadores tecnológicos, confrontando a utilidade e adequação dos seus recursos e } \\
\text { estratégias de aprendizado para os estudantes. Impulsionando questionamentos com } \\
\text { orientações que possibilitem novos conhecimentos para os estudantes quanto às } \\
\text { causas e efeitos das mudanças climáticas. Utilizar métodos de avaliação } \\
\text { inovadores: aplicar avaliações abertas que possibilitem uma pluralidade nas respostas }\end{array}$ \\
\hline
\end{tabular}

\footnotetext{
${ }^{1}$ https://education.minecraft.net

${ }^{2} \mathrm{https}: / /$ phet.colorado.edu/pt_BR/
} 


\begin{tabular}{|c|l|}
\hline & $\begin{array}{l}\text { dos estudantes, permitindo com que eles possam selecionar suas próprias ferramentas } \\
\text { e formas de apresentar suas ideias, aprimorando suas práticas e demonstrando o } \\
\text { aprendizado através de diversas maneiras (avaliação de pares, autoavaliação, } \\
\text { storyboard, portfólio, pitch, etc.). }\end{array}$ \\
\hline Soft Skill & $\begin{array}{l}\text { Consciência Social e Cultural: uso de tecnologias de forma responsável e ética pelo } \\
\text { estudante, estimulando a conscientização dele sobre problemas ambientais } \\
\text { antropológicos, seus impactos e as possibilidades de ação para mudar esta realidade. } \\
\text { Pensamento Crítico e Analítico: questionar os outros e a si mesmo e tomar decisões } \\
\text { sobre as temáticas abordadas. Resolução de Problemas: utilizar o pensamento } \\
\text { divergente para encontrar diferentes alternativas para resolver os problemas propostos } \\
\text { e o pensamento convergente para escolher a alternativa mais adequada ao contexto. }\end{array}$ \\
\hline Hard Skill & $\begin{array}{l}\text { Pensamento Computacional: usar decomposição, reconhecimento de padrões, } \\
\text { abstração e algoritmos, por meio da interação com os simuladores, viabilizando o } \\
\text { Discente } \\
\text { problema proposto. Solução de problemas de maneira criativa: saber usar a } \\
\text { criatividade para encontrar soluções fora do comum para solucionar o problema. }\end{array}$ \\
\hline Pedagogia & $\begin{array}{l}\text { Aprendizagem Baseada em Problemas: utilizar a necessidade de preservação da } \\
\text { biodiversidade e a degradação dos biomas naturais como problema base para que os } \\
\text { estudantes adquiram conhecimento sobre o tema e busquem soluçães de mitigação da } \\
\text { ação antrópica causadora de mudanças climáticas. Aprendizagem Lúdica: utilizar o } \\
\text { engajamento e motivação proporcionados pelo ambiente do jogo para o alcance do do } \\
\text { objetivo educacional, no qual os estudantes são imersos em atividades lúdicas para } \\
\text { resolução dos problemas propostos. }\end{array}$ \\
\hline
\end{tabular}

\subsection{Proposta 2: mudanças climáticas integradas à disciplina de Física}

Esta proposta usa a temperatura e a umidade do ar, visando: investigar as propriedades térmicas e o seu impacto ambiental; analisar concepções práticas em que é possível aplicar os conhecimentos básicos da termologia, estudando de que forma afeta as mudanças climáticas no decorrer do tempo; e confrontar a aplicação de ações técnicas com as noções dos fenômenos térmicos adquiridos para analisar as variações na quantidade de energia solar e umidade que provocam perturbações no clima terrestre. $\mathrm{O}$ educador pode, a partir das práticas realizadas, traçar um paralelo de situações em que se aplicam ou se manifestam os fenômenos térmicos no cotidiano dos estudantes, explanando o efeito térmico em cada caso e relacionando com a discussão sobre os percentuais de gases de efeito estufa que são emitidos, refletidos e absorvidos pela superfície da Terra e transformados em calor. Ainda, os educadores podem levantar temáticas como, por exemplo, os principais poluentes atmosféricos causadores do efeito estufa (queima de combustíveis fósseis, chaminés de fábricas, queimadas, etc.). A Tabela 2 apresenta os habilitadores da Educação 4.0 para a Proposta 2.

Tabela 2. Habilitadores da Educação 4.0 para a Proposta 2

\begin{tabular}{|c|l|}
\hline Habilitador & \multicolumn{1}{|c|}{ Descrição } \\
\hline \multirow{3}{*}{ Habilitadores } & $\begin{array}{l}\text { Internet das Coisas: utilizar sensores para monitorar umidade e temperatura do } \\
\text { ambiente, e visualizar os valores aferidos em um monitor serial. Para tanto, são } \\
\text { necessários: 1 placa Arduino com Cabo USB, 3 cabos jumper macho-fêmea e 1 } \\
\text { sensor de umidade e temperatura DHT11. Software Educativo: outra forma é usar } \\
\text { TinkerCAD ou Scratch for Arduino4. Nesse caso, é possível programar o teste } \\
\text { simulando a placa real, utilizando blocos de construção para desenvolver os } \\
\text { algoritmos sem a necessidade de conhecimento de uma linguagem de programação } \\
\text { específica. }\end{array}$ \\
\hline Habilitadores & $\begin{array}{l}\text { Melhorar a experiência educacional com o uso de tecnologias digitais: utilizar } \\
\text { tecnologias digitais para aumentar o interesse do estudante, aplicando o } \\
\text { Organizacionais } \\
\text { conhecimento num contexto visível e próximo do mundo real. Autonomia para } \\
\text { professores para inovar: autorizar os professores para tomarem decisões sobre } \\
\text { processos pedagógicos, atuando como projetistas do aprendizado. }\end{array}$ \\
\hline Competência & Ensinar a aprender a aprender: proporcionar aos estudantes uma contínua \\
\hline
\end{tabular}

\footnotetext{
${ }^{3}$ https://www.tinkercad.com/

$4 \mathrm{http}: / / \mathrm{s} 4 \mathrm{a} . \mathrm{cat} /$
} 


\begin{tabular}{|c|c|}
\hline Digital Docente & $\begin{array}{l}\text { [re]adaptação ao ambiente através do "aprender fazendo". Fornecer feedback } \\
\text { constante: acompanhar o processo e fornecer retorno constante, promover uma } \\
\text { perspectiva mais aproximada dos acontecimentos, e ajudar a solucionar os problemas } \\
\text { à medida que eles surgem. Saber qual tecnologia utilizar de acordo com a } \\
\text { necessidade: incrementar a experiência através da seleção de outros habilitadores } \\
\text { tecnológicos, promovendo questionamentos com propostas articuladoras que } \\
\text { agreguem conhecimento no combate às mudanças climáticas. }\end{array}$ \\
\hline $\begin{array}{l}\text { Soft Skill } \\
\text { Discente }\end{array}$ & $\begin{array}{l}\text { Criatividade: liberdade para fazer alterações e aprimoramentos na experiência e } \\
\text { inovar na forma de execução ou apresentação das soluções. Resolução de } \\
\text { Problemas: identificar e superar obstáculos que levem a melhoria das soluções de } \\
\text { tratamento das causas e impactos das mudanças no cotidiano. Trabalho em Equipe: } \\
\text { colaborar com outros estudantes, separar responsabilidades e contribuir com as ideias } \\
\text { alheias na construção da solução. }\end{array}$ \\
\hline $\begin{array}{l}\text { Hard Skill } \\
\text { Discente }\end{array}$ & $\begin{array}{l}\text { Pensamento Computacional: aperfeiçoar o raciocínio lógico, depurar erros e } \\
\text { aprimorar o pensamento lógico e sistemático de maneira natural enquanto entende o } \\
\text { problema e encontra a solução. }\end{array}$ \\
\hline Pedagogia & $\begin{array}{l}\text { Aprendizagem Orientada para a Ação: trabalhar com os estudantes para planejar e } \\
\text { desenvolver projetos de mitigação das causas e efeitos das mudanças climáticas, } \\
\text { capacitando-os para utilizarem os conhecimentos adquiridos como base para produzir } \\
\text { mudanças efetivas na sociedade. Aprendizagem Baseada em Projetos: trabalhar } \\
\text { com ações centradas nos estudantes, onde eles possam aprender sobre o assunto } \\
\text { por meio da experiência de procurar solucionar um problema real e ultrapassar o } \\
\text { ambiente da escola. }\end{array}$ \\
\hline
\end{tabular}

\section{Obtendo feedback}

Esta atividade iniciou com a elaboração de um vídeo de apresentação contendo as propostas especificadas anteriormente; e de um questionário eletrônico para avaliação. Em seguida, o vídeo e o questionário eletrônico foram disponibilizados para educadores (professores e pesquisadores da área de Educação).

\subsection{Avaliando as propostas de experiências de ensino-aprendizagem}

Esta atividade consistiu na visualização dos vídeos e avaliação dos habilitadores por meio do preenchimento do formulário eletrônico pelos 53 educadores participantes. A Figura 2 mostra o quantitativo (a) do perfil dos educadores com destaque para "Professor de Ensino Superior" (22) e "Professor de Ensino Fundamental" (15); e (b) do nível de formação avaliadores, com destaque para "Especialização" e "Mestrado" com 19 respostas cada. Em seguida, foi perguntado qual a área de atuação dos educadores, assim, sendo possível detalhar melhor o perfil deles. A Figura 3 mostra o quantitativo, com maior destaque para atuações em "Biologia" (12), "Física" (11) e "Tecnologia" (11).

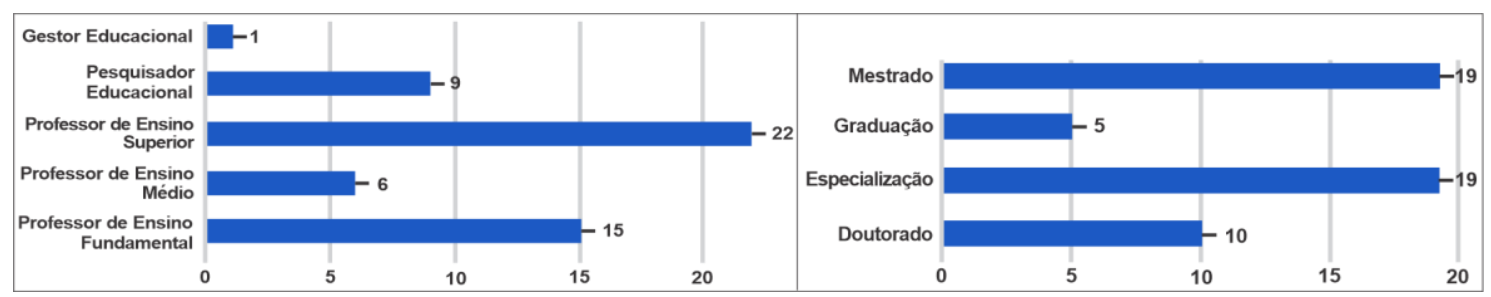

Figura 2 - (a) Perfil e (b) Nível de formação dos avaliadores.

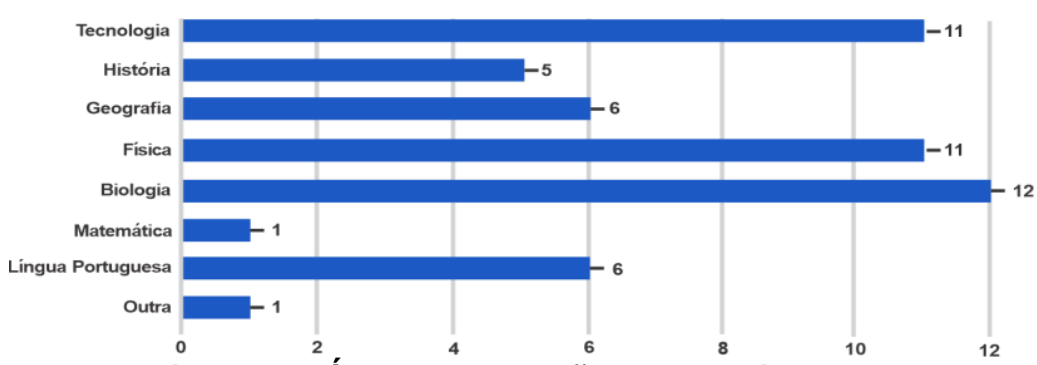

Figura 3 - Áreas de atuação dos avaliadores. 


\subsection{Analisando os resultados}

Esta atividade consistiu em analisar e discutir os resultados das avaliações dos habilitadores pelos educadores. Inicialmente, as respostas sobre as seguintes questões foram analisadas: (Q1) As propostas contribuem para elevar a conscientização e o conhecimento dos estudantes sobre as causas e efeitos das mudanças climáticas?; (Q2) As propostas contribuem com a formação de estudantes "digitais"?; e (Q3) O aprendizado de assuntos relacionados às mudanças climáticas é mais efetivo sendo integrado ao contexto de disciplinas tradicionais (Matemática, Física, História, etc.)?. Tais questões procuraram retratar a percepção dos avaliadores sobre a agregação de valor dos habilitadores no processo de ensino e aprendizagem, considerando a escala Likert de 1 - "discordo totalmente" a 5 - "concordo totalmente". A Figura 4 sintetiza a avaliação sobre as questões Q1, Q2 e Q3.

Segundo a UNESCO (2015), a educação é essencial para auxiliar os estudantes no entendimento e a lidar com as causas e impactos das mudanças climáticas, bem como para estimular as necessárias mudanças nas atitudes e hábitos para a assunção de estilos de vida mais sustentáveis. O resultado da avaliação da questão Q1 mostra que 88,7\% dos educadores concordam que as propostas contribuem para elevar a conscientização e o conhecimento dos estudantes sobre as mudanças climáticas.

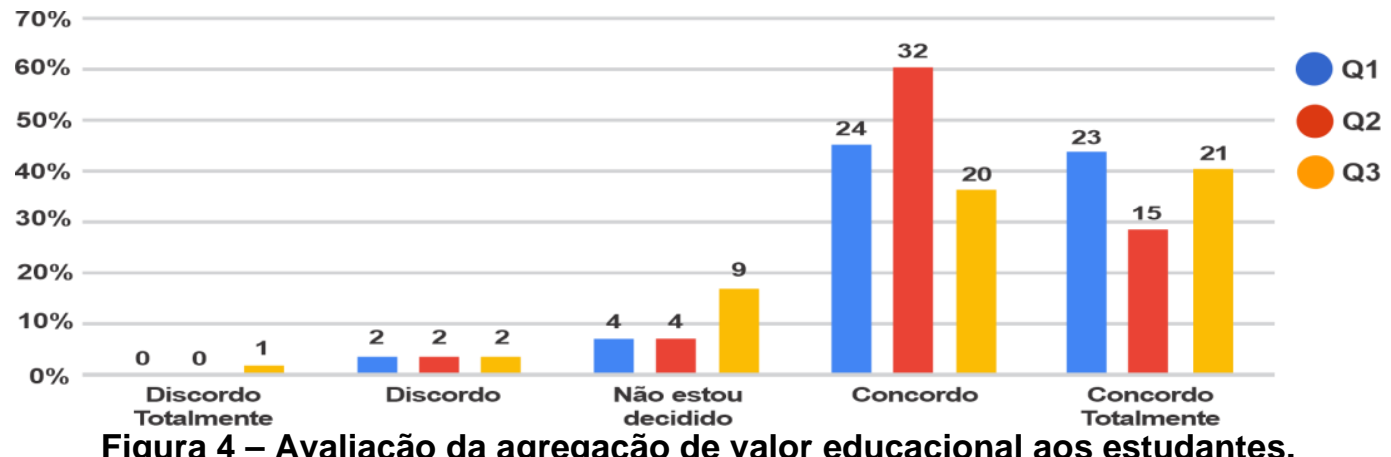

Figura 4 - Avaliação da agregação de valor educacional aos estudantes.

De acordo com a OECD (2018), as escolas necessitam preparar estudantes para trabalhos que ainda não foram criados, para tecnologias que ainda não foram inventadas, para resolver problemas que ainda não foram previstos. Para tanto, é necessário equipar os estudantes com as competências digitais para atender as exigências dos trabalhos emergentes da $4^{a}$ Revolução Industrial [WEF 2020]. O resultado da avaliação da questão Q2 mostra que $88,7 \%$ dos educadores concordam que as propostas contribuem com a formação de estudantes "digitais".

Segundo a UNESCO (2016), não se faz necessário um curso extracurricular para educar sobre as mudanças climáticas na escola, é possível e recomendado abranger questões propícias a esse tema em todas as disciplinas. $\mathrm{O}$ resultado da avaliação da questão Q3 mostra que 77,3\% dos educadores concordam que o aprendizado de assuntos relacionados às mudanças climáticas é mais efetivo sendo integrado no contexto de disciplinas tradicionais, como Matemática, Física, História, etc.

Em seguida, foram analisadas as respostas sobre: "quais habilidades, necessárias para o aprendizado e trabalho do século XXI, os habilitadores contribuem para equipar os estudantes com?". A Figura 5 apresenta o resultado, considerando que os avaliadores poderiam escolher mais de uma alternativa. 


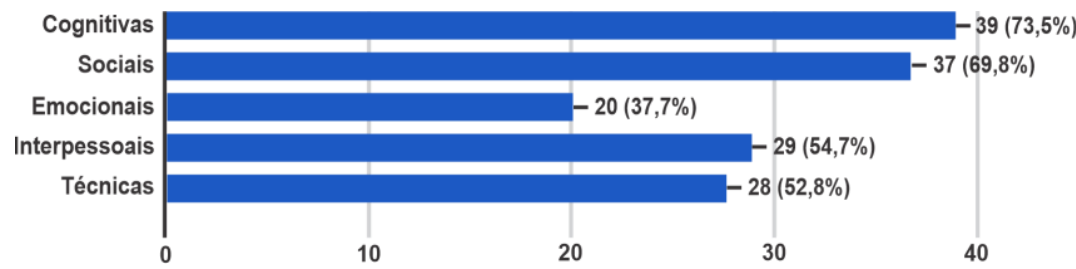

Figura 5 - Avaliação da agregação de habilidades do século XXI aos estudantes.

Para a OECD (2018), os estudantes precisarão empregar seus conhecimentos em cenários desconhecidos e em constante evolução, necessitando de uma gama extensa de habilidades cognitivas, sociais, emocionais, práticas e físicas. O resultado da avaliação (Figura 5) mostra que as propostas de experiências de ensino-aprendizagem contribuem para equipar os estudantes com habilidade do século XXI, com destaque para as habilidades cognitivas e sociais.

Por fim, foram analisadas as respostas sobre quais os benefícios que os habilitadores poderiam trazer para os professores. A Figura 6 apresenta os resultados, considerando que os avaliadores poderiam escolher mais de uma alternativa.

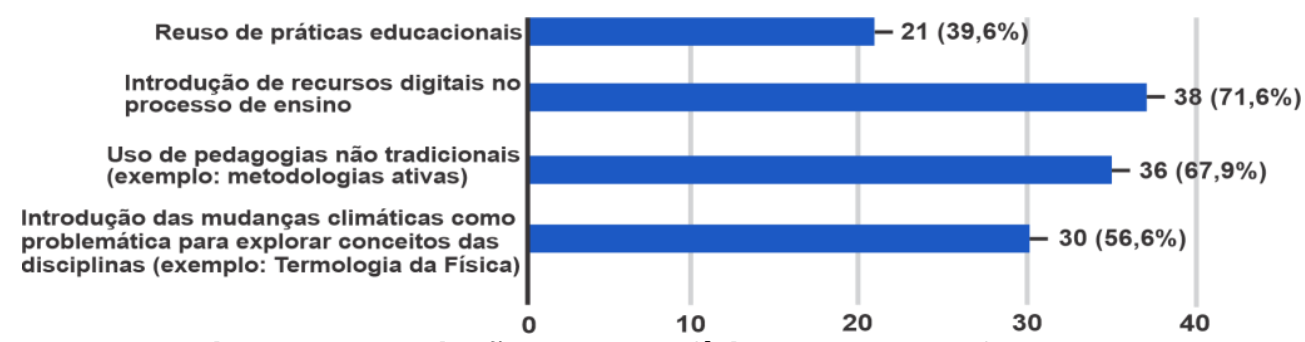

Figura 6 - Avaliação dos benefícios para os professores.

Segundo Schleicher (2016), os professores precisam utilizar tecnologias digitais no ensino e perceber o acelerado desenvolvimento dos campos de conhecimento. De acordo com a UNESCO (2015), em se tratando de mudanças climáticas, os professores necessitam de uma assimilação precisa do tema e conectar-se com questões mais abrangentes do desenvolvimento sustentável. O resultado da avaliação (Figura 6) mostra que as propostas trazem benefícios para os professores, com destaque para "introdução de recursos digitais no processo de ensino" e "uso de pedagogias não tradicionais".

\section{Discussões}

Esta avaliação dos habilitadores da Educação 4.0 permitiu um entendimento de que as novas abordagens pedagógicas são fundamentais para tornar a educação relevante e atrativa para os estudantes. As habilidades sociais e técnicas necessitam ser alteradas conforme a esfera social do século XXI, sendo essencial que sejam ensinadas e aprendidas de forma envolvente para que possam ser adaptadas pelos estudantes em cenários que se transformam constantemente.

De acordo com [Oliveira e de Souza 2021], para adequadamente abordar as principais adversidades da atualidade, dentre elas as mudanças climáticas, é importante haver um processo de transformação digital na educação. Desse modo, os resultados desse estudo sugerem que os habilitadores para transformação digital em direção à Educação 4.0 podem ajudar a atender às necessidades educacionais exigidas pela $4^{\mathrm{a}}$ Revolução Industrial, principalmente no que diz respeito às competências digitais dos professores e às soft e hard skills essenciais para o aprendizado dos estudantes.

Nas práticas sugeridas neste estudo, um dos diferenciais e contribuições é a possibilidade de educar e conscientizar os estudantes sobre as causas e efeitos das mudanças climáticas em forte conexão com disciplinas básicas, ao mesmo tempo que é 
utilizado de modo holístico as tecnologias digitais. Ainda, as experiências utilizam-se de pedagogias inovadoras para fomentar competências digitais nos professores e habilidades técnicas, interpessoais, sociais e cognitivas nos estudantes, as habilidades do século XXI.

\section{Conclusões e Trabalhos Futuros}

Este trabalho apresentou a especificação e avaliação de duas propostas de uso dos habilitadores de transformação digital em direção à Educação 4.0 propostos por [De Sousa Oliveira e de Souza 2020]. Ambas as propostas têm como propósito usar a temática "mudanças climáticas" como base para a transferência (ensino) e aquisição (aprendizado) de conhecimentos das disciplinas de: (i) Biologia, por meio da simulação da degradação de biomas naturais causados pelas perturbações do clima; e (ii) Física, por meio da termologia para explicar as consequências do aumento da temperatura no meio ambiente. Entre os diferenciais e contribuições destacam-se: (i) educar e conscientizar sobre as causas e efeitos das mudanças climáticas em forte conexão com disciplinas básicas; (ii) uso holístico de tecnologia digital, visão organizacional, pedagogia, competência digital docente e habilidades técnicas, interpessoais, sociais e cognitivas discente, como estratégia para a transformação digital da educação. Ressalta-se, ainda, que a transformação digital na educação tende a ser ainda mais necessária em um cenário de convivência ou pós-pandemia do COVID-19.

O feedback dos 53 educadores avaliadores indica sua concordância quanto ao valor educacional do método proposto: eleva a conscientização e o conhecimento dos estudantes sobre as causas e efeitos das mudanças climáticas; contribui com a formação de estudantes "digitais"; o aprendizado de assuntos relacionados às mudanças climáticas pode ser mais efetivo sendo integrado ao contexto de disciplinas tradicionais; contribui em proporcionar aos estudantes habilidades cognitivas e sociais; e introduz recursos digitais no processo de ensino, bem como uso de pedagogias não tradicionais pelos professores.

De fato, os resultados obtidos a partir do feedback dos educadores apontam que os habilitadores podem dar apoio, habilitar e direcionar iniciativas de transformação digital na educação, ao mesmo tempo em que podem contribuir com a educação e conscientização dos estudantes sobre as causas e efeitos das mudanças climáticas em forte conexão com disciplinas básicas.

Entre as perspectivas para a continuidade da pesquisa destacam-se o desenvolvimento de uma ferramenta baseada nos habilitadores para transformação digital em direção à Educação 4.0, com o objetivo de apoiar a criação e reuso de experiências de ensino-aprendizagem; e a execução das propostas apresentadas em um contexto educacional real.

\section{Agradecimentos}

Os autores agradecem às agências de fomento brasileiras - Fundação de Amparo à Pesquisa do Estado de São Paulo (FAPESP) sob o processo no 2018 / 26636-2 e Coordenação de Aperfeiçoamento de Pessoal de Nível Superior - Brasil (CAPES) Código Financeiro 001.

\section{Referências}

De Sousa Oliveira, K. K., and de Souza, R. A. C. (2020). Habilitadores da transformação digital em direção à Educação 4.0. RENOTE, 18(1). 
Frerich, S., Meisen, T., Richert, A., Petermann, M., Jeschke, S., Wilkesmann, U. and Tekkaya, A. E. (2016). Engineering education 4.0. Cham: Springer International Publishing.

Gonçalves, A. S., Lopes, M. and Reis, D. (2019). Simulador para prevenção e mitigação aos riscos de desastres utilizando realidade virtual. In Anais do Workshop de Informática na Escola (Vol. 25, No. 1, pp. 1239-1243).

INEP. Instituto Nacional de Estudos e Pesquisas Educacionais Anísio Teixeira. (2012) "Matriz de Referência ENEM". Brasília.

OECD. Organisation for Economic Co-operation and Development. (2018). The future of education and skills: Education 2030. OECD Education 2030. OECD Publishing, Paris.

OECD. Organisation for Economic Co-operation and Development. (2019). Trends Shaping Education 2019, OECD Publishing, Paris.

Oliveira, K. K. D. S., and de Souza, R. A. (2021). Digital Transformation towards Education 4.0. Informatics in Education. doi:10.15388/infedu.2022.13.

Oliveira, K. K., and de Souza, R. (2020). Mudanças climáticas na educação: um levantamento das práticas, ferramentas e tecnologias digitais. In Anais do XI Workshop de Computação Aplicada à Gestão do Meio Ambiente e Recursos Naturais (pp. 151-160). SBC.

ONU. Organização das Nações Unidas. (2015). "Transformando Nosso Mundo: A Agenda 2030 para o Desenvolvimento Sustentável”. https://nacoesunidas.org/wpcontent/uploads/2015/10/agenda2030-pt-br.pdf.

Rosenbloom, D. and Markard, J. (2020). A COVID-19 recovery for climate. Science, 368, 447

Schleicher, A. (2016), Teaching Excellence through Professional Learning and Policy Reform: Lessons from Around the World, International Summit on the Teaching Profession, OECD Publishing, Paris.

UNESCO. United Nations Educational, Scientific and Cultural Organization. (2013). Climate Change in the Classroom: UNESCO Course for Secondary Teachers on Climate Change Education for Sustainable Development. Paris: UNESCO.

UNESCO. United Nations Educational, Scientific and Cultural Organization. (2020). "COVID-19 Educational Disruption and Response". https://pt.unesco.org/covid19/educationresponse/, Maio.

UNESCO. United Nations Educational, Scientific and Cultural Organization. (2016). Getting climate ready: a guide for schools on climate action and the whole-school approach. UNESCO Publishing, Paris.

UNESCO. United Nations Educational, Scientific and Cultural Organization. (2015). Not Just Hot Air: Putting Climate Change Education into Practice. UNESCO Publishing, Paris.

UNESCO. United Nations Educational, Scientific and Cultural Organization. (2019). The ethical challenges of climate change. UNESCO Publishing, Paris.

WEF. World Economic Forum. (2016). The future of jobs: Employment, skills and workforce strategy for the fourth industrial revolution. In Global Challenge Insight Report, World Economic Forum. Cologny/Geneva, Switzerland

WEF. World Economic Forum. (2020). Schools of the Future. Defining New Models of Education for the Fourth Industrial Revolution. In Platform for Shaping the Future of the New Economy and Society. Cologny/Geneva, Switzerland. 